\title{
Dimensionamento hidráulico de drenos subterrâneos: Proposta de equação geral
}

\author{
Wilson Deniculi ${ }^{1}$, Mauro A. Martinez ${ }^{1} \&$ Demetrius D. da Silva ${ }^{1}$ \\ 1 DEA/UFV. CEP 36570-000, Viçosa, MG. Fone: (31) 3899-2729. E-mails: deniculi@ufv.br (Foto), mmauro@ufv.br e \\ david@ufv.br \\ Protocolo $121-23 / 8 / 2002$ - Aprovado em 21/5/2003
}

\begin{abstract}
Resumo: Objetivou-se, através deste trabalho, desenvolver uma equação geral para o dimensionamento hidráulico de drenos subterrâneos, visto que na literatura disponível só existem equações obtidas a partir de valores de rugosidade do tubo e de temperatura da água específicas. Para o equacionamento do problema, admitiu-se que a vazão coletada aumenta linearmente a partir do início do dreno, considerado fechado na sua extremidade inicial. As equações apresentadas podem ser usadas para o dimensionamento de drenos subterrâneos com quaisquer características hidráulicas e qualquer equação válida para condutos livres em regime permanente e uniforme, adaptando-se às características de rugosidade do tubo e do fluido em escoamento. Desta forma, os cálculos feitos com a equação proposta produzem resultados mais próximos da realidade do problema.
\end{abstract}

Palavras-chave: drenos subterrâneos, equação geral, vazão

\section{A general equation for the hydraulic design of subsurface drains}

\begin{abstract}
This research had the objective of presenting a general equation for the hydraulic design of subsurface drains, since the equations available in the literature were obtained using specified drain roughness coefficient and water temperature. To develop the equation, it was considered that the drain is closed at the beginning and that the flow rate increases linearly along the entire drain length. The developed equation may be used to design subsurface drains having any specified hydraulic characteristics and using any uniform steady state equation for open channel design, which can be adapted to different drain roughness coefficients and fluid characteristics. Therefore, the calculations done using this equation bring more realistic results.
\end{abstract}

Key words: subsurface drain, general equation, flow rate

\section{INTRODUÇÃO}

O dimensionamento de condutos livres (canais), em condições de escoamento permanente e uniforme, é feito com o auxílio de equações empíricas, sendo que todas elas podem ser agrupadas em uma única do tipo, obedecendo-se ao Sistema Internacional de Unidades (SI):

$$
\mathrm{Q}=\mathrm{CA}(\mathrm{R} I)^{\mathrm{m}}
$$

em que:
Q - vazão
C - fator de resistência
A - área molhada do canal
$\mathrm{R}$ - raio hidráulico do canal
I - declividade de fundo do canal, adimensional
$\mathrm{m}$ - expoente da declividade, adimensional

O raio hidráulico $(\mathrm{R})$ é uma dimensão resultante da relação entre a área molhada (A) e o perímetro molhado (P) do canal.

O fator de resistência é uma função do raio hidráulico e da natureza das paredes do canal. Este fator, juntamente com o expoente da declividade, tem sido alvo de pesquisas.

Estudos realizados por Manning (1891) permitiram concluirse que o expoente da declividade $(\mathrm{m})$ pode ser considerado igual a 0,5 e o fator de resistência (C), segundo Henderson (1980), pode ser calculado por:

$$
\mathrm{C}=\mathrm{R}^{1 / 6} \mathrm{n}^{-1}
$$

sendo $\mathrm{n}$ conhecido como fator de Manning, tabelado de acordo com a natureza das paredes do canal.

Em outro estudo, Bazin (1897) verificou que o valor de $\mathrm{m}$ também poderia ser considerado igual a 0,5 , porém o valor de C, conforme Chow (1986), deveria ser calculado: 


$$
C=\frac{87 \sqrt{R}}{y+\sqrt{R}}
$$

sendo y conhecido como coeficiente de Bazin, tabelado de acordo com a natureza das paredes do canal.

Considerando-se também a fórmula universal de perda de carga, pode-se calcular $\mathrm{C}$ por:

$$
C=\sqrt{\frac{8 g}{f}}
$$

sendo $\mathrm{g}=9,81 \mathrm{~m} \mathrm{~s}^{-2}$ e f o coeficiente de atrito, adimensional.

Os sistemas de drenagem podem ser feitos por meio de drenos abertos (valetas) e drenos subterrâneos (cobertos). Estes últimos apresentam a vantagem de facilitar operações de transporte e mecanização, evitando a construção de pontes e outras estruturas necessárias no caso de utilização de valetas (Millar, 1978).

O dimensionamento de drenos abertos é realizado com a aplicação das equações apresentadas anteriormente, o que não ocorre para o caso dos drenos subterrâneos, tendo em vista que a vazão escoada varia desde o início do dreno até o seu final.

Em razão disto, diferentes equações experimentais são apresentadas para o dimensionamento de drenos subterrâneos, o que leva o projetista a dúvidas na escolha de uma ou outra equação.

Neste trabalho pretende-se apresentar um fator de correção único, de tal forma que a Eq. 1 também possa ser utilizada para o dimensionamento de drenos subterrâneos simplificando, deste modo, a tomada de decisão por parte do usuário.

\section{DESENVOLVIMENTO DA EQUAÇÃO GERAL}

No desenvolvimento da equação geral para o dimensionamento hidráulico de drenos subterrâneos, foram consideradas as seguintes premissas:

a) os drenos subterrâneos são tratados como condutos livres;

b) o escoamento é considerado permanente e uniforme;

c) o escoamento pode ocorrer em qualquer forma de seção (circular, retangular etc.), desde que fechada;

d) a vazão de montante é nula $\left(\mathrm{Q}_{\mathrm{M}}=0\right)$;

e) o escoamento é fluvial;

f) a vazão coletada por unidade de comprimento do dreno (q) é constante, enquanto a vazão do dreno (Q) varia linearmente com o seu comprimento;

g) à exceção dos parâmetros adimensionais, já destacados na Eq. 1, os demais obedecem ao Sistema Internacional de Unidades (SI).
A contribuição linear da vazão é uma simplificação utilizada no dimensionamento hidráulico de drenos, assim como a distribuição linear da vazão ao longo da tubulação é usada para o dimensionamento de condutos forçados dotados de múltiplas saídas (Silvestre, 1979 e Deniculi, 2002).

Tendo em vista que no escoamento permanente e uniforme a declividade de fundo do canal (I) é igual à perda de carga unitária $(\mathrm{J})$ pode-se reescrever a Eq. 1 como:

$$
\mathrm{I}=\mathrm{J}=\frac{\mathrm{h}_{\mathrm{f}}}{\mathrm{L}}=\frac{1}{(\mathrm{C} \mathrm{A})^{1 / \mathrm{m}}} \frac{\mathrm{Q}^{1 / \mathrm{m}}}{\mathrm{R}}
$$

em que $h_{f}$ representa a perda de carga total ao longo do comprimento do dreno.

Fazendo:

$$
\beta=\frac{1}{\mathrm{R}(\mathrm{C} \mathrm{A})^{1 / \mathrm{m}}}
$$

pode-se escrever a Eq. 5 como:

$$
\mathrm{h}_{\mathrm{f}}=\beta \mathrm{LQ} \mathrm{Q}^{1 / \mathrm{m}}
$$

Para o desenvolvimento da equação geral de dimensionamento hidráulico considerou-se um dreno subterrâneo fechado na extremidade inicial, conforme Figura 1, levando-se em conta as premissas mencionadas anteriormente.

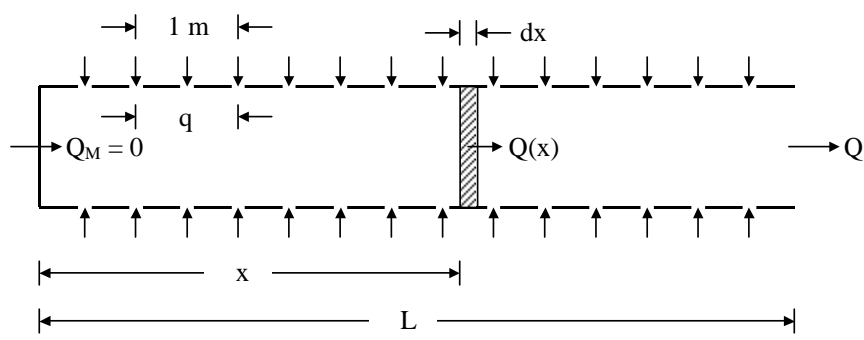

Figura 1. Dreno subterrâneo fechado na extremidade inicial

Aplicando-se a Eq. 7 ao trecho elementar (dx) da figura anterior, percorrido pela vazão $\mathrm{Q}(\mathrm{x})$ tem-se:

$$
\mathrm{dh}_{\mathrm{f}}=\beta \mathrm{Q}_{(\mathrm{x})}^{1 / \mathrm{m}} \mathrm{dx}
$$

Levando-se em consideração a Figura 1, verifica-se que:

$$
\mathrm{Q}(\mathrm{x})=\mathrm{q} \mathrm{x}
$$

$\mathrm{e}$

$$
\mathrm{Q}=\mathrm{q} \mathrm{L}
$$

em que q é a vazão coletada a cada metro de dreno, $\mathrm{m}^{3} \mathrm{~s}^{-1} \mathrm{~m}^{-1}$.

Substituindo-se a Eq. 9 na Eq. 8 e integrando-a ao longo de todo o comprimento $\mathrm{L}$, tem-se: 


$$
\mathrm{h}_{\mathrm{f}}=\beta \mathrm{q}^{1 / \mathrm{m}} \int_{0}^{\mathrm{L}} \mathrm{x}^{1 / \mathrm{m}} \mathrm{dx}
$$

equação esta que, após realizada a sua integração e substituindo-se qL por Q, Eq. 10, pode ser escrita como:

$$
\mathrm{h}_{\mathrm{f}}=\frac{\beta}{1 / \mathrm{m}+1} \mathrm{~L} \mathrm{Q}^{1 / \mathrm{m}}
$$

Comparando-se a Eq. 12, válida para condutos com entrada de água ao longo do comprimento L (caso de drenos subterrâneos), com a Eq. 7, válida para condutos sem entrada de água ao longo de L, verifica-se que elas diferem apenas no fator $\left(1_{\mathrm{m}}+1\right)^{-1}$.

Escrevendo-se a Eq. 12 numa forma mais adequada à dos condutos livres, tem-se:

$$
\frac{\mathrm{h}_{\mathrm{f}}}{\mathrm{L}}=\mathrm{J}=\mathrm{I}=\frac{\beta}{1 / \mathrm{m}+1} \mathrm{Q}^{1 / \mathrm{m}}
$$

ou

$$
Q=\left[\left(\frac{1 / m+1}{\beta}\right) I\right]^{m}
$$

ou, ainda, pela substituição da Eq. 6 em 14:

$$
\mathrm{Q}=\left({ }^{1}{ }_{\mathrm{m}}+1\right)^{\mathrm{m}} \mathrm{CA}(\mathrm{R} \mathrm{I})^{\mathrm{m}}
$$

sendo esta a equação geral proposta para drenos subterrâneos, operando com qualquer forma de seção (circular, retangular etc) e vazão de montante nula $\left(\mathrm{Q}_{\mathrm{M}}=0\right)$. Nota-se que esta equação difere da Eq. 1 apenas no coeficiente $\left({ }_{\mathrm{m}}+1\right)^{\mathrm{m}}$ significando que qualquer equação válida para condutos livres pode ser usada para o dimensionamento de drenos subterrâneos, desde que se leve em conta o referido coeficiente.

\section{EQUAÇÃO PARA SEÇÕES CIRCULARES}

Na prática, a seção mais utilizada para drenos subterrâneos apresenta a forma circular.

Adequando-se, portanto, a Eq. 15 para drenos cobertos circulares operando a plena seção, chega-se a:

$$
Q=(1 / m+1)^{m} \frac{\pi C}{4^{m+1}} D^{m+2} I^{m}
$$

tendo-se em conta que:

$$
\mathrm{A}=\frac{\pi \mathrm{D}^{2}}{4}
$$

e

$$
\mathrm{R}=\frac{\mathrm{D}}{4}
$$

sendo D o diâmetro interno do dreno, $\mathrm{m}$.

Particularizando-se a Eq. 16 e se tendo em vista, respectivamente, as experiências de Manning $\left(\mathrm{m}=0,5\right.$ e $\left.\mathrm{C}={ }^{1 /} \mathrm{R}^{1 / 6}\right)$ e a fórmula universal $(\mathrm{m}=0,5$ e $\mathrm{C}=\sqrt{8 \mathrm{~g} / \mathrm{f}})$ pode-se escrever que:

$$
\mathrm{Q}=\frac{0,54}{\mathrm{n}} \mathrm{D}^{2,667} \mathrm{I}^{0,5}
$$

e

$$
Q=\frac{6,02}{\sqrt{f}} D^{2,5} I^{0,5}
$$

Nos casos particulares de drenos corrugados $(n=0,0141)$ e drenos lisos ( $\mathrm{f}=0,3164 \mathrm{Re}^{-0,25}$, sendo Re o número de Reynolds) as Eqs. 19 e 20 podem ser reescritas, respectivamente, como:

$$
\mathrm{Q}=38,3 \mathrm{D}^{2,667} \mathrm{I}^{0,5}
$$

e

$$
\mathrm{Q}=111,7 \mathrm{D}^{2,714} \mathrm{I}^{0,571}
$$

Beltrán (1986) apresenta as seguintes equações para dimensionamento hidráulico de drenos corrugados e lisos, respectivamente:

$$
\mathrm{Q}=38,3 \mathrm{D}^{2,667} \mathrm{I}^{0,5}
$$

e

$$
Q=89 D^{2,714} I^{0,571}
$$

A Eq. 21, demonstrada no presente trabalho, é igual à Eq. 23 apresentada por Beltrán (1986) o qual aborda o tema tendo em vista apenas a equação de Manning aplicada a condutos de seção circular. Ressalta-se, entretanto, que a equação geral proposta neste trabalho (Eq. 15) da qual se derivou a Eq. 21, pode ser aplicada a qualquer seção e a qualquer equação válida para condutos livres.

A Eq. 22 difere da Eq. 24 apresentada por Beltrán (1986) em função do fato de se ter usado, para o cálculo de f, um coeficiente igual a $0,3164\left(f=0,3164 \mathrm{Re}^{-0,25}\right)$ no lugar de 0,40 ( $\mathrm{f}=0,40 \mathrm{Re}^{-0,25}$ ) e uma temperatura ambiente da água de $20^{\circ} \mathrm{C}$ no lugar de $10^{\circ} \mathrm{C}$, temperatura esta mais realística para as condições ambientais brasileiras.

Um procedimento semelhante, tendo em conta as experiências de Bazin, onde $m=0,5$ e $C=87 \sqrt{R}(y+\sqrt{R})^{-1}$ permite 
reescrever a Eq. 16, válida tanto para tubos lisos como para corrugados, na forma:

$$
\mathrm{Q}=59,1 \frac{\mathrm{D}^{3}}{2 \mathrm{y}+\mathrm{D}^{0,5}} \mathrm{I}^{0,5}
$$

\section{SIMULAÇÃO DE VAZÕES}

$\mathrm{Na}$ Tabela 1 são apresentadas as vazões calculadas em $\mathrm{L} \mathrm{h}^{-1}$, para tubos lisos e corrugados, usando-se as equações propostas por Beltrán (1986) e as desenvolvidas neste trabalho.

Tabela 1. Vazões, em L h ${ }^{-1}$, obtidas com o uso das Eqs. 21, 22 e 25 e com a equação proposta por Beltran (1986) para tubos lisos e corrugados

\begin{tabular}{lcccc}
\hline \multirow{2}{*}{ Equações } & \multicolumn{4}{c}{ Tubos $(\mathrm{cm})$} \\
\cline { 2 - 3 } & \multicolumn{2}{c}{ Lisos $(\mathrm{D}=8)^{*}$} & \multicolumn{2}{c}{ Corrugados $(\mathrm{D}=7,2)^{*}$} \\
\cline { 2 - 3 } \cline { 5 - 5 } & $\mathrm{I}=0,001$ & $\mathrm{I}=0,002$ & $\mathrm{I}=0,001$ & $\mathrm{I}=0,002$ \\
\hline Eq. Beltrán & 1,82 & 2,70 & 1,08 & 1,53 \\
Eq. 21 & --- & -- & 1,08 & 1,53 \\
Eq. 22 & 2,28 & 3,39 & --- & --- \\
Eq. 25 $(\mathrm{y}=0,060)^{* *}$ & 2,38 & 3,36 & --- & --- \\
Eq. 25 $(\mathrm{y}=0,200)^{* *}$ & --- & --- & 1,04 & 1,48 \\
\hline * Diâmetros comerciais & & & &
\end{tabular}

** Valores de y obtidos de Giles (1978)

Analisando-se os dados da Tabela 1, verifica-se que, tanto para tubos lisos quanto para corrugados, as vazões obtidas através das Eqs. 22 e 25, propostas neste trabalho, apresentaram valores bem próximos. Ao se analisar, entretanto, os resultados obtidos com a equação de Beltran para tubos lisos, observa-se uma diferença média de $22 \%$ quando comparados aos resultados calculados com as Eqs. 22 e 25. Ressalta-se que esta diferença decorre do uso, nas Eqs. 22 e 25, de coeficientes de rugosidade e temperatura da água diferentes, tornando o dimensionamento com o uso dessas equações mais flexível, adaptando-se às características de rugosidade do tubo e do fluído em escoamento, o que não é possível com o uso da equação de Beltran, a qual é fundamentada numa rugosidade do tubo e temperatura da água.

No caso dos tubos corrugados, a equação de Beltran apresentou resultados iguais aos da Eq. 21, em função do fato de se ter adotado o mesmo valor do coeficiente de rugosidade “n”, por não se encontrar, na literatura, diferentes valores para este. Os resultados das duas equações foram bem próximos daqueles obtidos com a Eq. 25 e, de maneira semelhante ao que ocorre para tubos lisos, as Eqs. 21 e 25 permitem maior flexibilidade no dimensionamento de tubos de drenagem com diferentes características hidráulicas.

\section{CONCLUSÕES}

1. As equações propostas no presente trabalho podem ser usadas para o dimensionamento hidráulico de drenos subterrâneos com qualquer forma geométrica e qualquer equação válida para condutos livres em regime permanente $\mathrm{e}$ uniforme, tornando o dimensionamento mais flexível.

2. As equações propostas adaptam-se às características de rugosidade do tubo e do fluido em escoamento, resultando em cálculos mais confiáveis para as condições brasileiras.

3. Para tubos lisos, a equação de Beltran subestimou a capacidade máxima de transporte de água do dreno em comparação com as equações propostas no trabalho.

\section{LITERATURA CITADA}

Bazin, H. Étude d'une nouvelle formule pour calculer le débit des cannaux découverts. Annales des ponts et chaussés, v.14, 1897. 50p.

Beltrán, J.M. Drenaje agrícola. 1 ed. Madrid: Editora Secretaria General Técnica, 1986. 283p.

Chow, V.T. Open channel hydraulic. 3 ed. New York: McGrawHill Book Company, 1986. 680p.

Denículi, W.; Silva, D.D.; Oliveira, R.A. Hidráulica de condutos perfurados. 1 ed. Viçosa: Editora UFV, 2002. 68p.

Giles, P.L. Mecânica dos fluidos e hidráulica. São Paulo: McGraw-Hill do Brasil, 1978. 401p.

Henderson, F.M. Open channel flow. 3 ed. New York: Macmillan Publishing Co, 1980. 522p.

Manning, R. On the flow of water in open channels and pipes. Transactions Institution of Civil Engineers of Ireland, Dublin, v.20, 1891, 47p.

Millar, A.A. Drenagem de terras agrícolas: bases agronômicas. 1 ed. São Paulo: McGraw-Hill do Brasil, 1978. 276p.

Silvestre, P. Hidráulica geral. Livros Técnicos e Científicos. 1 ed. Rio de Janeiro: Editora S.A, 1979. 316p. 